\title{
Reduction in nosocomial infections during the COVID-19 era: a lesson to be learned
}

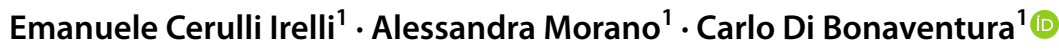

Received: 6 October 2020 / Accepted: 9 November 2020 / Published online: 19 November 2020

(c) Italian Society of Surgery (SIC) 2020

Dear Editor-in-chief,

We read with great interest the manuscript by Losurdo et al. that reported a reduced rate of surgical site infections (SSIs) during the COVID-19 pandemic as compared with previous years, possibly in relation to the implementation of hygienic measures and the systematic use of personal protective equipment (PPE) among healthcare workers and patients [1].

In a recent paper published by our group, we investigated whether the increase in hygienic measures during the pandemic determined a decrease in hospital-acquired infections (HAIs) as compared with the previous year [2]. The study was conducted in a COVID-free unit to avoid the possible confounding impact of a COVID-19 coinfection. After adjusting for other variables, we found a significant decrease in HAIs and antibiotic prescriptions in our unit, which we think was largely attributable to the huge increase in hygienic measures.

Similarly, Ponce-Alonso et al. recently found an almost $70 \%$ reduction in Clostridioides difficile infections in a tertiary-care hospital in Spain during the COVID-19 outbreak [3]. These authors also ascribed this reduction to the stricter hygienic measures adopted during the recent pandemic.

In light of these findings, we strongly believe that a serious debate regarding the need to extend the reinforcement of infection prevention and control (including the systematic use of PPE) beyond the COVID-19 pandemic, is particularly urgent. Indeed, the high burden of HAIs in terms of mortality and public spending is well known and has been highlighted by multiple reports published by EU agencies [4]. Among HAIs, SSIs represent the most common infection in low-income countries, and one of the most frequent in high-income countries [4]. When considering the EU and USA together, an estimated 140,000 deaths are directly

Carlo Di Bonaventura

c_dibonaventura@yahoo.it

1 Sapienza University of Rome, Rome, Italy attributable to HAIs [5]. Moreover, it is well known that HAIs also contribute to other deaths, perhaps as many as 110,000 in the EU alone [5].

Despite countless calls for action by international agencies during the last years to improve programs for infection prevention and control worldwide [4], these calls have largely been unheeded, and a rapid improvement in the implementation of these programs seemed unlikely. However, the recent COVID-19 pandemic has clearly taught us a lesson: in the midst of a health emergency, the majority of developed countries succeeded in rapidly planning and implementing more efficient hygiene protocols in their hospitals.

It would be a huge mistake to waste the opportunity offered by COVID-19 to finally take a step forward in improving infection prevention and control.

\section{Compliance with ethical standards}

Conflict of interest None of the authors have any conflicts of interest to disclose.

Research involving human participants and/or animals No research involving human participants and/or animals was conducted for the purpose of this paper.

Informed consent Informed consent was not applicable to this article.

\section{References}

1. Losurdo P, Paiano L, Samardzic N et al (2020) Impact of lockdown for SARS-CoV-2 (COVID-19) on surgical site infection rates: a monocentric observational cohort study. Updates Surg $14: 1-9$

2. Cerulli Irelli E, Orlando B, Cocchi E et al (2020) The potential impact of enhanced hygienic measures during the COVID-19 outbreak on hospital-acquired infections: a pragmatic study in neurological units. J Neurol Sci 418:117111 
3. Ponce-Alonso M, Sáez de la Fuente J, Rincón-Carlavilla A et al (2020) Impact of the coronavirus disease 2019 (COVID-19) pandemic on nosocomial Clostridioides difficile infection. Infect Control Hospital Epidemiol. https://doi.org/10.1017/ice.2020.454

4. Suetens C, Latour K, Kärki T et al (2018) Prevalence of healthcare-associated infections, estimated incidence and composite antimicrobial resistance index in acute care hospitals and longterm care facilities: results from two European point prevalence surveys, 2016 to 2017. Euro Surveill 23:180051
5. World Health Organization The burden of health care-associated infection worldwide. Available at https://www.who.int/gpsc/count ry_work/summary_20100430_en.pdf

Publisher's Note Springer Nature remains neutral with regard to jurisdictional claims in published maps and institutional affiliations. 